\title{
Zehn Jahre therapeutische milde Hypo- thermie - Bullen- oder Bärenmarkt?
}

\author{
Gerhard Jorch
}

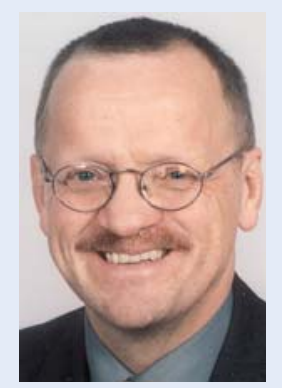

Gerhard Jorch
Korrespondenzadresse Prof. Dr. Gerhard Jorch Universitätsklinikum Magdeburg Universitätskinderklinik Leipziger Str. 44 39112 Magdeburg gerhard.jorch@med. ovgu.de $\mathbf{v}$ or 10 Jahren wurden 2 große Studien publiziert, die umgehend zu einer Empfehlung der milden Hypothermie in den ILCOR-Richtlinien für die Behandlung bewusstloser Patienten nach Kreislaufstillstand führten [1,3,4]. Bereits kurz darauf wurde aufgrund vorläufiger und endgültiger Studienergebnisse die milde Hypothermie auch für die Behandlung von (reifen) Neugeborenen nach schwerer perinataler Asphyxie empfohlen $[2,5]$.

Zumindest für die genannten Patientengruppen überwog der neuroprotektive Nutzen nicht nur statistisch signifikant, sondern auch klinisch relevant die systemischen Nebenwirkungen der Therapie, sodass eine $15 \%$ ige Steigerung der Heilungsrate bzw. ein zusätzlich geheilter Patient pro 7 behandelte Patienten resultierten. Eine derart hoher Vorteil wird bei den meisten Studien neu eingeführter Medikamente nicht erreicht. Deshalb warnten von Beginn an erfahrene Intensivmediziner vor einer zu großen Euphorie, der später in einem Blues enden könne wie bei so manchem eingeführten Medikament.

10 Jahre später ist aber die erwartete „Baisse“ nicht eingetreten, sondern es dominiert weiter ein umsichtiger „Bullenmarkt“, um dieses aus der Finanzwelt uns mittlerweile geläufige Begriffspaar zu verwenden. Die Therapie ist fast flächendeckend eingeführt, wird mit Augenmaß betrieben und hat die Intensivmedizin bei Patienten vom Lebensanfang bis zum Erwachsenenalter um eine wichtige neue therapeutische Option bereichert. In diesem Heft stellen 2 Autorengruppen den derzeitigen Stand der therapeutischen Hypothermie im Kindes- und im Erwachsenalter dar.

Für die Indikation „Hypoxisch-ischämische Enzephalopathie des Reifgeborenen“ liegt mittlerweile eine ungewöhnlich hohe Evidenz aus 6 Multicenterstudien vor. Die Therapie ist verbindlicher Standard in der Neonatologie. Die ERC-Leitlinien von 2010 legen die Eckpunkte der Therapie fest [6]. Die Evidenz für die Indikation „Bewusstlosigkeit nach Kreislaufstillstand im Kindesalter" ist derzeit weniger gut belegt. Die
Anwendung der milden Hypothermie bei dieser Indikation ist also im Einzelfall gerechtfertigt, aber nicht in jedem Fall zwingend geboten. Die Erkenntnisse aus Studien zur Indikation „Schädel-Hirn-Trauma“ im Kindesalter deuten derzeit eher darauf hin, dass die Nebenwirkungen der Therapie den Nutzen überwiegen. Deshalb muss der Einsatz der Hypothermie bei dieser Indikation bis auf Weiteres auf kontrollierte und durch eine Ethikkommission konsentierte Studien beschränkt bleiben. Bei erwachsenen Patienten ist die Indikation „Bewusstlosigkeit nach Kreislaufstillstand“ etabliert. Besonders günstig scheint das Verhältnis von Nutzen zu Nebenwirkungen beim Herzstillstand durch initial tachykarde Rhythmusstörungen außerhalb des Krankenhauses zu sein. Die ERC-Empfehlung von 2010 hat im Gegensatz zur Empfehlung von 2005 die Indikation nun auf "nicht defribillierbare Herzrhythmen“ erweitert [7]. Für andere Indikationen wie z.B. „Polytrauma“, „Schädel-Hirn-Trauma“, ,ischämischer Schlaganfall“, „hepatische Enzephalopathie und Enzephalomeningitis" gilt, dass die Anwendung der milden Hypothermie möglichst nur im Rahmen von klinischen Studien erfolgen sollte.

Fazit Die Einführung der milden Hypothermie in die Intensivmedizin darf weiter als Erfolgsgeschichte betrachtet werden. Patientengefährdungen durch überschwängliche Indikationsausweitungen wurden bisher mit Augenmaß vermieden. Dieses Beispiel zeigt, dass auch heute noch innovative und hochwirksame Therapien weitgehend ohne industrielle Sponsoren eingeführt werden können und deren Abstinenz nicht zwingend zu einem Bärenmarkt führt.

Die Literatur zu diesem Beitrag finden Sie unter http://dx.doi.org/10.1055/s-0032-1309424. 